Review Article

\title{
Comparison of Minimally Invasive Percutaneous Plate Osteosynthesis and Open Reduction Internal Fixation on Proximal Humeral Fracture in Elder Patients: A Systematic Review and Meta-Analysis
}

\author{
Wei Zhao, ${ }^{1}$ Yuhui Zhang, ${ }^{2}$ Dongni Johansson, ${ }^{1}$ Xingyu Chen, ${ }^{1}$ \\ Fang Zheng, ${ }^{1}$ and Liangman $\mathrm{Li}^{1}$ \\ ${ }^{1}$ Department of Orthopaedics, No. 1 Hospital of China Medical University, Shenyang 110001, China \\ ${ }^{2}$ Department of Dermatology, No. 1 Hospital of China Medical University, Shenyang 110001, China \\ Correspondence should be addressed to Liangman Li; liliangman1@126.com
}

Received 11 February 2017; Accepted 23 May 2017; Published 15 June 2017

Academic Editor: Georg Gradl

Copyright (C) 2017 Wei Zhao et al. This is an open access article distributed under the Creative Commons Attribution License, which permits unrestricted use, distribution, and reproduction in any medium, provided the original work is properly cited.

\begin{abstract}
Objective. The study aims to compare minimally invasive percutaneous plate osteosynthesis (MIPO) and open reduction internal fixation (ORIF) in the treatment of proximal humeral fracture in elder patients. Method. PubMed, Medline, EMbase, Ovid, Cochrane Library, China National Knowledge Infrastructure (CNKI), Wangfang, and VIP Database for Chinese Technical Periodicals were searched to identify all relevant studies from inception to October 2016. Data were analyzed with Cochrane Collaboration's Review Manage 5.2. Results. A total of 630 patients from 8 publications were included in the systematic review and meta-analysis. The pooled results showed that MIPO was superior to ORIF in the treatment of proximal humeral fracture in elder patients. It was reflected in reducing blood loss, operation time, postoperative pain, or fracture healing time of the surgery and in improving recovery of muscle strength. Concerning complications, no significant difference was seen between MIPO and ORIF. Conclusion. The MIPO was more suitable than ORIF for treating proximal humeral fracture in elder patients.
\end{abstract}

\section{Introduction}

Proximal humeral fracture is one of the most frequent osteoporotic fractures in the elderly people. The incidence of proximal humeral fracture is increasing with population ages and traffic accidents in urban areas gradually. And women are affected more frequently than men [1].

Therapeutic regimen of proximal humeral fracture should be formulated according to the Neer classification of proximal humeral fracture, which is based on 4 anatomical segments of the proximal humerus and whether these segments are displaced or not $[1,2]$. Nondisplaced fractures are commonly treated by conservative treatment; in contrast, displaced fractures are often treated by operations including open reduction internal fixation (ORIF), intramedullary device fixation, minimally invasive plate osteosynthesis (MIPO), or hemiarthroplasty [3].

Of all options, ORIF is a very commonly used method for proximal humeral fracture. Nevertheless, previous studies have shown that there were a raising number of complications with ORIF, including reposition failure, malunion, infection, internal loosening, prosthesis implantation failure, and humerus head vascular necrosis [4]. Recently, minimally invasive plate osteosynthesis (MIPO) has become increasingly popular in the management of fractures [5]. Early clinical studies indicated that MIPO could limit soft tissue injury, reduce operation time, and relieve destruction in blood supply. However, the optimal surgical approach for proximal fracture remains controversial. This systematic review aims to compare MIPO and ORIF for proximal 
TABLE 1: Characteristics of the included studies.

\begin{tabular}{|c|c|c|c|c|c|}
\hline Reference & $\begin{array}{c}\text { Number of patients } \\
\text { MIPO/ORIF }\end{array}$ & $\begin{array}{c}\text { Gender } \\
\text { Male/female }\end{array}$ & $\begin{array}{c}\text { Mean age } \\
\text { MIPO/ORIF }\end{array}$ & $\begin{array}{l}\text { Follow-up } \\
\text { (Months) }\end{array}$ & $\begin{array}{c}\text { Operative } \\
\text { Type }\end{array}$ \\
\hline Zhao et al. & $37 / 31$ & $26 / 42$ & $71.3 / 71.5$ & 12 & Neer: $2,3,4$ \\
\hline Song & $39 / 39$ & $23 / 55$ & 64.2 & 12 & Neer: $2,3,4$ \\
\hline Shang et al. & $24 / 54$ & $19 / 59$ & $61.6 / 60.0$ & 12 & Neer: $2,3,4$ \\
\hline Wang et al. & $20 / 20$ & $14 / 26$ & $69.6 / 69.7$ & 12 & Neer: 2, 3 \\
\hline Lin et al. & $43 / 43$ & $28 / 58$ & $63 / 61$ & 12 & $\mathrm{AO}: \mathrm{A}, \mathrm{B}, \mathrm{C}$ \\
\hline Liu et al. & $47 / 51$ & $43 / 55$ & $72.8 / 49.9$ & 12 & Neer: 3,4 \\
\hline Liu et al. & $33 / 42$ & $28 / 47$ & $47.3 / 49.1$ & 12 & Neer: 2, 3 \\
\hline Götz et al. & $61 / 46$ & $32 / 75$ & $65 / 67.6$ & 12 & $\mathrm{AO}: \mathrm{A}, \mathrm{B}, \mathrm{C}$ \\
\hline
\end{tabular}

Note. Neer's terminology of four-segment classification of displaced fractures and fracture-dislocations relates pattern of displacement (Neer: 2, two-part; Neer: 3, three-part; or Neer: 4, four-part) and key segment displaced. The AO classification is based on the severity of the fracture and the likely disruption to the vascularity of the proximal humerus, including three broad types of fracture. Type A fractures are extra-articular and unifocal, type B fractures are extraarticular and bifocal, and type $\mathrm{C}$ fractures are articular.

humeral fracture in the elder to provide clinical guidance for surgeons.

\section{Materials and Methods}

2.1. Search Strategy. PubMed, Medline, EMbase, Ovid, Cochrane Library, China National Knowledge Infrastructure (CNKI), Wangfang, and VIP Database for Chinese Technical Periodicals were searched to identify relevant studies that compared MIPO with ORIF for the treatment of proximal humeral fractures from inception to October 2016. The search strategy combined the following terms: "proximal humeral/humerus fracture", "internal fixation/ORIF”, "minimally invasive/MIPO". Languages and types of articles were not restricted. A manual search was supplemented by verifying the references listed in the key publications.

2.2. Study Selection. An overall literature search was performed and relevant studies were screened independently by two reviewers (Wei Zhao, Dongni Johansson). Qualified studies were selected based on the following criteria: (1) design of study: randomized controlled study (RCT) or nRCT; (2) age of patients: $\geq 45$ years old with proximal humeral fracture; (3) intervention: MIPO and ORIF; (4) at least one of following data having been recorded in articles: intraoperative blood loss, operation time, postoperation pain, complications, fracture healing time, and functional outcomes.

Exclusion criteria are as follows: (1) animal model experiment; (2) the cases of patient being lower than 10; (3) having no relevant data that could be extracted from articles; (4) duplicate publication.

2.3. Data Extraction and Quality Assessment. Relevant data were extracted independently by two reviewers (Wei Zhao, Dongni Johansson). The designed form included the following information: first author's name, publication year, number of patients, mean age, follow-up time, operative type, intraoperative blood loss, operation time, postoperation pain, complications, time of fracture healing, and functional outcomes.
The quality assessment of included studies was independently performed and crosschecked by two reviewers. Disagreements were resolved by discussing with a senior researcher (Liangman Li).

2.4. Statistical Analysis. The meta-analysis was conducted using Review Manage 5.2 provided by Cochrane Collaboration. Inverse-variance test was applied for continuous variables and the Mantel-Haenszel test was applied to examine dichotomous variables. The weighted mean difference (WMD) was used to calculate continuous variables across studies that were measured in the same scale. Different scales of continuous variables were combined and calculated by standard mean differences (SMD). Dichotomous variables were carried out by using odds ratio (OR). All data were reported with WMD, SMD, or OR and associated $95 \%$ confidence intervals (CI). The heterogeneity between studies was tested by both Chi-square test and $I$-squared test $\left(I^{2}\right)$. $P<0.1$ or $I^{2}>50 \%$ was considered as a high heterogeneity between studies. If significant heterogeneity was present $\left(P<0.1, I^{2}>50 \%\right)$, a random-effect model was selected. A fixed-effect model was performed when the significant heterogeneity was absent across studies.

\section{Results}

3.1. Literature Search and Study Characteristics. A total of 1853 relevant studies were retrieved. After removal of duplicates by titles, 1749 articles were further screened. After careful identification, 20 studies were assessed by full-text. Finally, 8 articles met inclusion criteria and were included in the systematic review [3, 4, 6-11]. Selection progress of studies was shown in Figure 1. A total of 630 patients with proximal humeral fracture were involved, including 304 patients treated by MIPO and 326 patients treated by ORIF. Characteristics of patients were listed in Table 1.

3.2. Quality Assessment of Included Studies. As all the included studies were nRCT, the quality assessment was conducted by methodological index for nonrandomized 


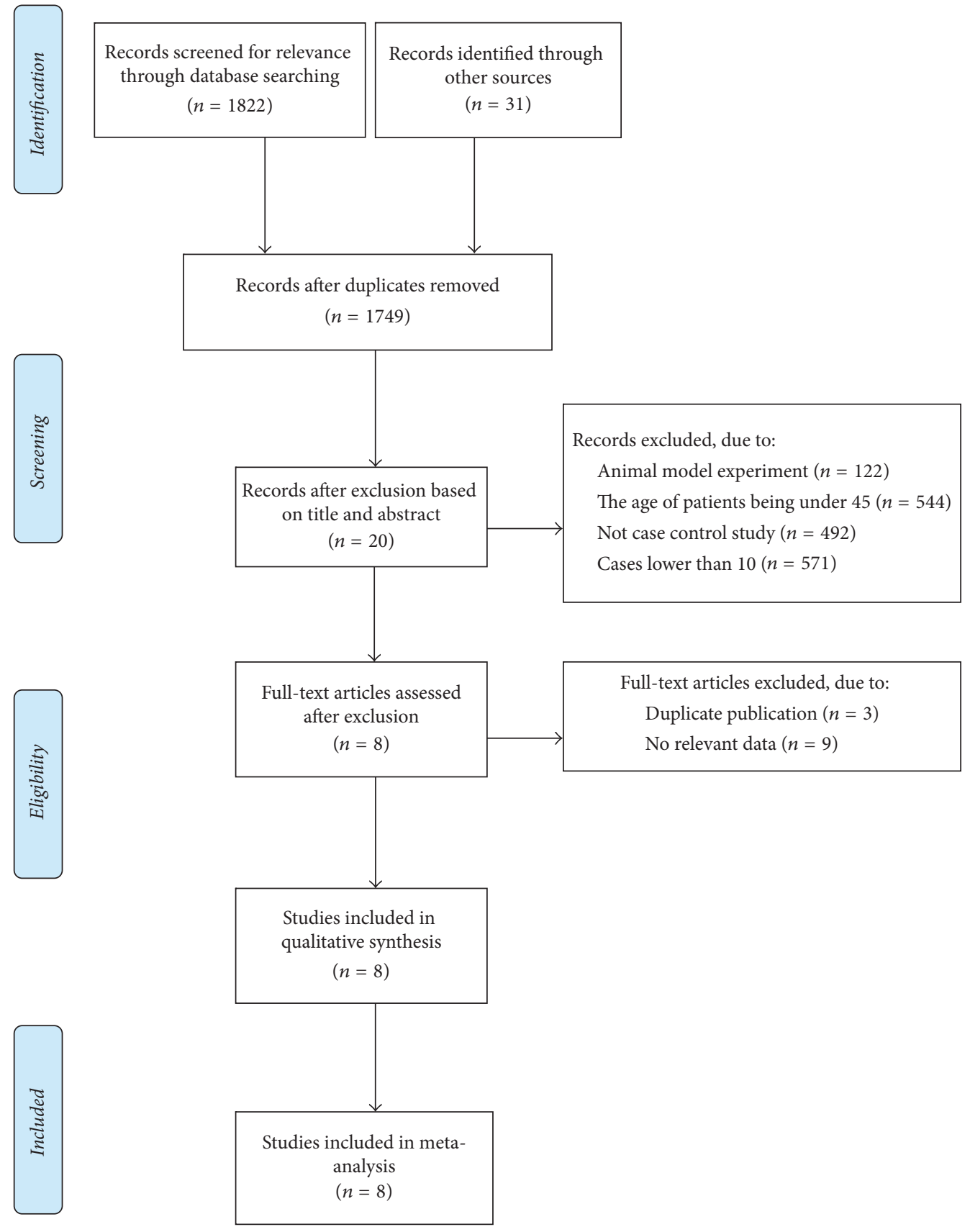

FIgURE 1: Flow diagram demonstrating those studies which were processed for inclusion.

studies (MINORS). Methodologic items were as follows: (1) a clearly stated aim; (2) inclusion of consecutive patients; (3) prospective collection of data; (4) end points appropriate to the aim of the study; (5) unbiased assessment of the study endpoint; (6) follow-up period appropriate to the aim of the study; (7) loss to follow-up, which is less than 5\%; (8) prospective calculation of the study size; (9) an adequate control group; (10) contemporary groups; (11) baseline equivalence of groups; (12) adequate statistical analyses. The items were scored as "0" (not reported), "1" (reported but inadequate), or "2" (reported and adequate). The global ideal score for comparative studies was 24 [12]. The score over 12 was regarded as high quality. The quality of included studies was presented in Table 2.

\subsection{Comparison of MIPO and ORIF on \\ Proximal Humeral Fracture}

3.3.1. Intraoperative Blood Loss and Operation Time. Of all the 8 studies, 6 studies which involved 445 cases and all the 8 studies which involved 630 cases, respectively, provided the data on intraoperative blood loss and operation time. There 
TABLE 2: MINORS appraisal scores for the included studies.

\begin{tabular}{|c|c|c|c|c|c|c|c|c|c|c|c|c|c|}
\hline \multirow{2}{*}{ Study } & \multicolumn{12}{|c|}{ Methodological items } & \multirow{2}{*}{ Total } \\
\hline & 1 & 2 & 3 & 4 & 5 & 6 & 7 & 8 & 9 & 10 & 11 & 12 & \\
\hline Zhao et al. & 2 & 1 & 0 & 1 & 0 & 2 & 2 & 0 & 1 & 2 & 1 & 2 & 14 \\
\hline Song & 2 & 2 & 0 & 2 & 0 & 2 & 1 & 0 & 2 & 2 & 2 & 2 & 17 \\
\hline Shang et al. & 2 & 1 & 0 & 2 & 0 & 2 & 1 & 0 & 2 & 2 & 2 & 2 & 16 \\
\hline Wang et al. & 2 & 2 & 0 & 2 & 0 & 2 & 2 & 0 & 2 & 2 & 1 & 2 & 17 \\
\hline Lin et al. & 2 & 2 & 0 & 2 & 0 & 2 & 1 & 0 & 2 & 2 & 2 & 2 & 17 \\
\hline Liu et al. & 2 & 2 & 0 & 2 & 0 & 2 & 2 & 0 & 1 & 2 & 1 & 2 & 16 \\
\hline Liu et al. & 2 & 1 & 0 & 2 & 0 & 2 & 1 & 0 & 2 & 2 & 2 & 2 & 16 \\
\hline Götz et al. & 2 & 2 & 0 & 2 & 0 & 2 & 2 & 0 & 2 & 2 & 2 & 2 & 18 \\
\hline
\end{tabular}

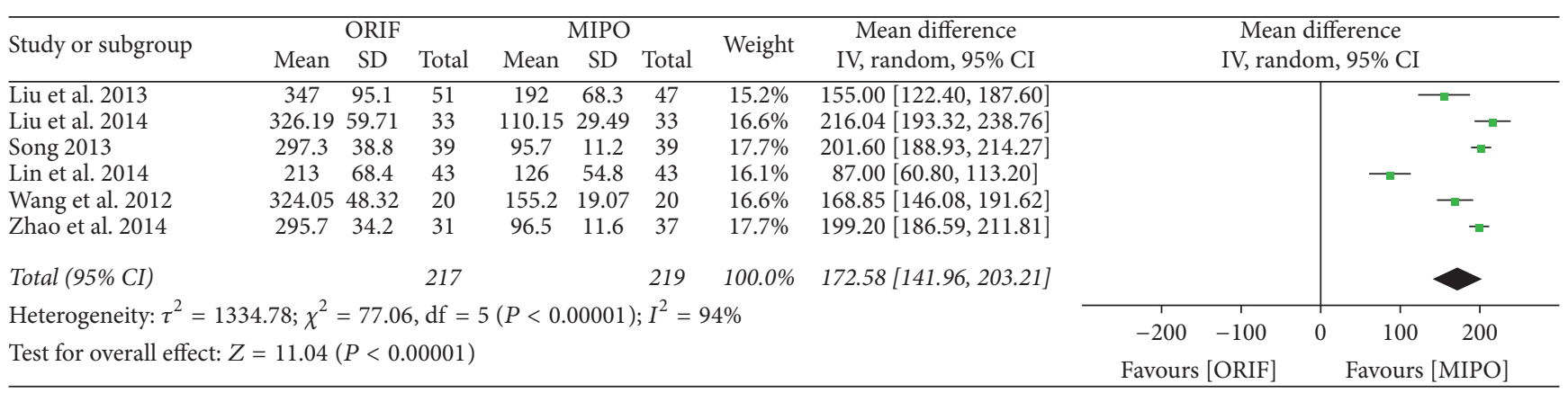

Figure 2: Forest plot of weighted mean difference (WMD) and 95\% confidence intervals (CI) for intraoperative blood loss.

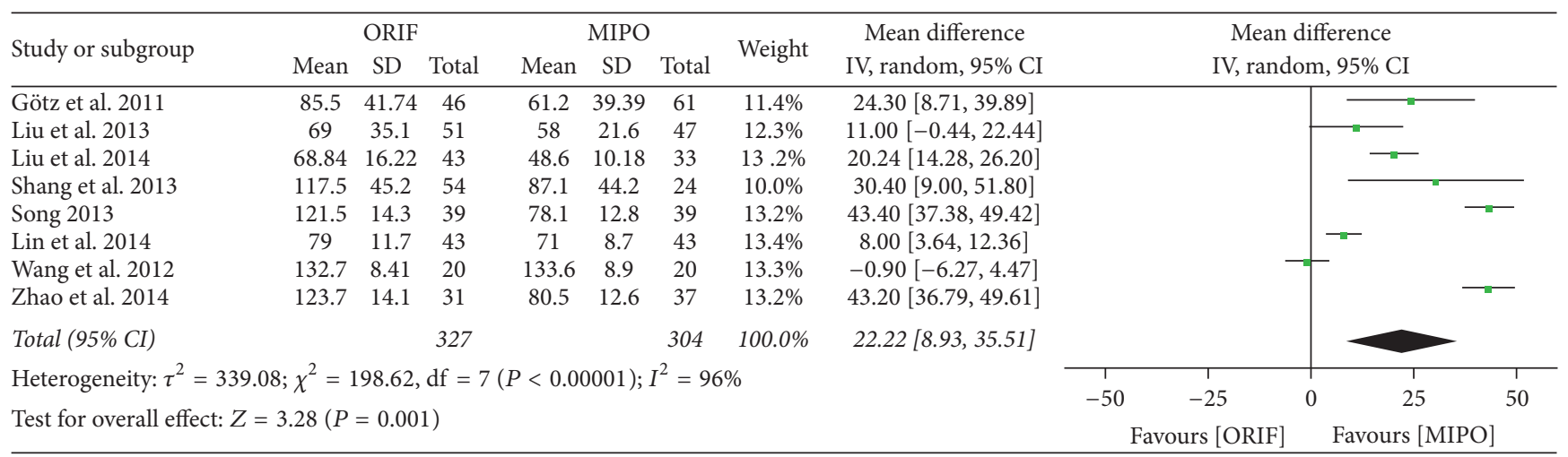

FigURE 3: Forest plot of weighted mean difference (WMD) and 95\% confidence intervals (CI) for operation time.

was a significant heterogeneity across studies (intraoperative blood loss: $P<0.00001, I^{2}=94 \%$; operation time: $P<$ $0.00001, I^{2}=96 \%$ ), and thus the random-effect models were applied. The meta-analysis indicated that MIPO was superior to ORIF in whatever intraoperative blood loss (WMD = $172.58 ; 95 \%$ CI, 141.96 to 203.21 ) or operation time (WMD = 22.22; $95 \% \mathrm{CI}, 8.93$ to 35.51 ) (Figures 2 and 3 ).

3.3.2. Postoperative Pain. Visual analog scale (VAS) was used to evaluate short-term postoperative pain in 1 week after surgery. And Constant-Murley score was used to evaluate long-term postoperative pain 6 to 12 months after surgery. Of all included studies, 3 studies provided data on VAS and 4 studies used Constant-Murley score. Statistical analysis of
VAS score showed a high homogeneity across studies $(P=$ $\left.0.88, I^{2}=0 \%\right)$, and thus the fixed-effect model was used for meta-analysis (Figure 4). However, the Constant-Murley score showed a significant heterogeneity across studies $(P<$ $\left.0.00001, I^{2}=92 \%\right)$, and therefore the random-effect model was performed. The meta-analysis in both VAS score $(\mathrm{SMD}=$ $0.56 ; 95 \%$ CI, 0.26 to 0.86 ) and Constant-Murley score (SMD $=1.28 ; 95 \%$ CI, 0.33 to 2.23 ) showed a better outcome treated by MIPO than by ORIF after surgery. Comparing to ORIF, MIPO effectively reduced postoperative pain (Figures 4 and 5).

3.3.3. Complications. Complications were available in 5 studies involving 437 patients. A significant heterogeneity was 


\begin{tabular}{|c|c|c|c|c|c|c|c|c|c|c|c|}
\hline \multirow{2}{*}{ Study or subgroup } & \multicolumn{3}{|c|}{ ORIF } & \multicolumn{3}{|c|}{ MIPO } & \multirow{2}{*}{ Weight } & \multirow{2}{*}{$\begin{array}{l}\text { Std. mean difference } \\
\text { IV, fixed, 95\% CI }\end{array}$} & \multirow{2}{*}{\multicolumn{3}{|c|}{$\begin{array}{l}\text { Std. mean difference } \\
\text { IV, fixed, 95\% CI }\end{array}$}} \\
\hline & Mean & $\mathrm{SD}$ & Total & Mean & $\mathrm{SD}$ & Total & & & & & \\
\hline Liu et al. 2014 & 3.26 & 0.8 & 42 & 2.88 & 0.78 & 33 & $41.4 \%$ & $0.48[0.01,0.94]$ & & - & \\
\hline Shang et al. 2013 & 5.5 & 0.8 & 24 & 5.1 & 0.6 & 54 & $36.9 \%$ & $0.59[0.10,1.08]$ & & - & \\
\hline Wang et al. 2012 & 5.31 & 2.22 & 20 & 3.83 & 2.09 & 20 & $21.7 \%$ & $0.67[0.03,1.31]$ & & & \\
\hline Total (95\% CI) & & & 86 & & & 107 & $100.0 \%$ & $0.56[0.26,0.86]$ & & & \\
\hline \multirow{2}{*}{\multicolumn{8}{|c|}{$\begin{array}{l}\text { Heterogeneity: } \chi^{2}=0.27, \mathrm{df}=2(P=0.88) ; I^{2}=0 \% \\
\text { Test for overall effect: } Z=3.70(P=0.0002)\end{array}$}} & -4 & -2 & 0 & 2 \\
\hline & & & & & & & & & Favours [ORIF] & Favou & rs [MIPO] \\
\hline
\end{tabular}

FIGURE 4: Forest plot of standard mean difference (SMD) and 95\% confidence intervals (CI) for postoperative pain of visual analog scale score.

\begin{tabular}{|c|c|c|c|c|c|c|c|c|c|c|c|}
\hline \multirow{2}{*}{ Study or subgroup } & \multicolumn{3}{|c|}{ MIPO } & \multicolumn{3}{|c|}{ ORIF } & \multirow{2}{*}{ Weight } & \multirow{2}{*}{$\begin{array}{l}\text { Std. mean difference } \\
\text { IV, random, } 95 \% \text { CI }\end{array}$} & \multirow{2}{*}{\multicolumn{3}{|c|}{$\begin{array}{l}\text { Std. mean difference } \\
\text { IV, random, } 95 \% \text { CI }\end{array}$}} \\
\hline & Mean & $\mathrm{SD}$ & Total & Mean & $\mathrm{SD}$ & Total & & & & & \\
\hline Liu et al. 2013 & 14.5 & 2.5 & 47 & 12.7 & 2.3 & 51 & $26.1 \%$ & $0.74[0.33,1.16]$ & & \multicolumn{2}{|l|}{ - } \\
\hline Liu et al. 2014 & 13.76 & 0.71 & 33 & 12.19 & 0.77 & 42 & $25.0 \%$ & $2.09[1.52,2.66]$ & & \multirow{3}{*}{$f=-$} & -- \\
\hline Lin et al. 2014 & 13.3 & 2.8 & 43 & 12.8 & 3.2 & 43 & $26.0 \%$ & $0.16[-0.26,0.59]$ & & & \\
\hline Wang et al. 2012 & 34.65 & 2.38 & 20 & 29.38 & 2.11 & 20 & $22.8 \%$ & $2.30[1.48,3.11]$ & & & $=-$ \\
\hline Total (95\% CI) & & & 143 & & & 156 & $100.0 \%$ & $1.28[0.33,2.23]$ & & & \\
\hline \multicolumn{8}{|c|}{ Heterogeneity: $\tau^{2}=0.85 ; \chi^{2}=39.91, \mathrm{df}=3(P<0.00001) ; I^{2}=92 \%$} & -4 & -2 & 0 & 2 \\
\hline \multicolumn{8}{|c|}{ Test for overall effect: $Z=2.65(P=0.008)$} & & Favours $[\mathrm{M}$ & \multicolumn{2}{|c|}{ Favours [ORIF] } \\
\hline
\end{tabular}

FIGURE 5: Forest plot of standard mean difference (SMD) and 95\% confidence intervals (CI) for postoperative pain of Constant-Murley score.

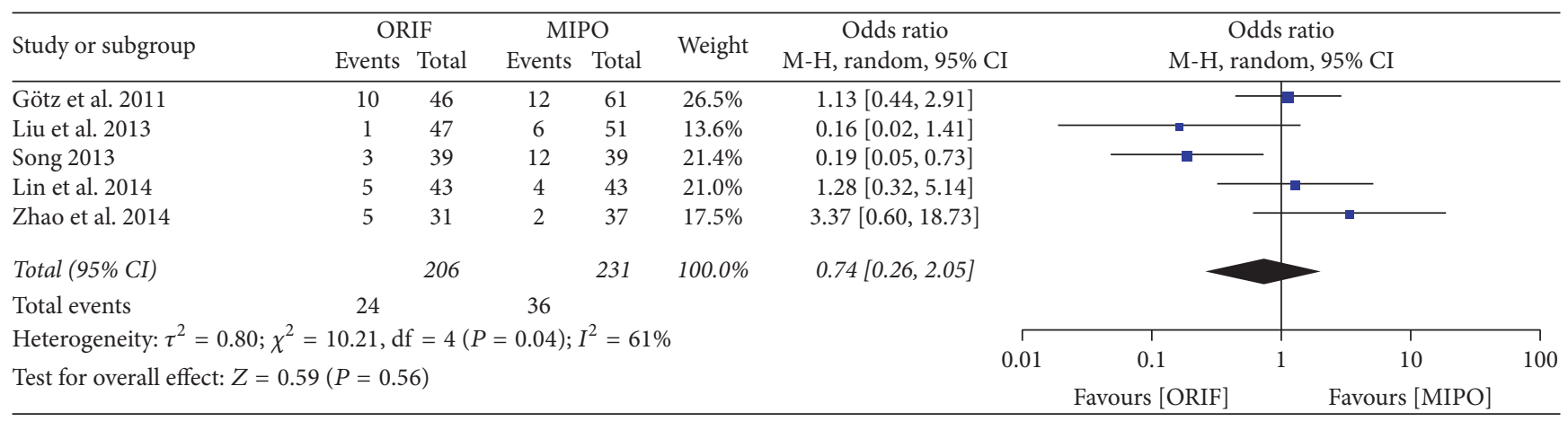

Figure 6: Forest plot of odds ratio (OR) and 95\% confidence intervals (CI) for complications.

seen across studies $\left(P=0.04, I^{2}=61 \%\right)$, and so the randomeffect model was used for meta-analysis. The pooled results showed that no statistical difference was found between ORIF and MIPO treatment $(\mathrm{OR}=0.74 ; 95 \% \mathrm{CI}, 0.26$ to 2.05$)$ (Figure 6).

3.3.4. Fracture Healing Time. Fracture healing time was reported in 3 studies, and the result showed that there was a significant heterogeneity among studies $\left(P=0.0003, I^{2}=\right.$ $87 \%)$. The meta-analysis conducted by random-effect model indicated that MIPO for proximal humeral fracture had a shorter time of fracture healing compared with ORIF (SMD $=0.86 ; 95 \% \mathrm{CI}, 0.10$ to 1.63 ) (Figure 7 ).

3.3.5. Functional Outcomes. Four articles provided functional outcomes with Constant-Murley score 6 to 12 months after surgery. A significant heterogeneity was seen across studies ( $\left.P=0.008, I^{2}=75 \%\right)$, and so the random-effect model was used for meta-analysis. The results of overall functional outcomes showed that no significant difference existed between ORIF and MIPO (SMD $=-0.27 ; 95 \% \mathrm{CI}$, -0.71 to 0.17 ) (Figure 8 ). However, MIPO had a better muscle strength of functional outcome $(\mathrm{SMD}=0.65 ; 95 \% \mathrm{CI}, 0.34$ to 0.95 ) from 6 to 12 months after surgery, indicating that MIPO could improve recovery of muscle strength compared to ORIF (Figure 9).

\section{Discussion}

To our knowledge, this is the first systematic review comparing MIPO and ORIF for the treatment of proximal humerus fracture. The present meta-analysis suggested that MIPO surgical techniques had apparent advantages in intraoperative blood loss, operation time, and fracture healing time compared to ORIF, whereas there was no statistically significant 


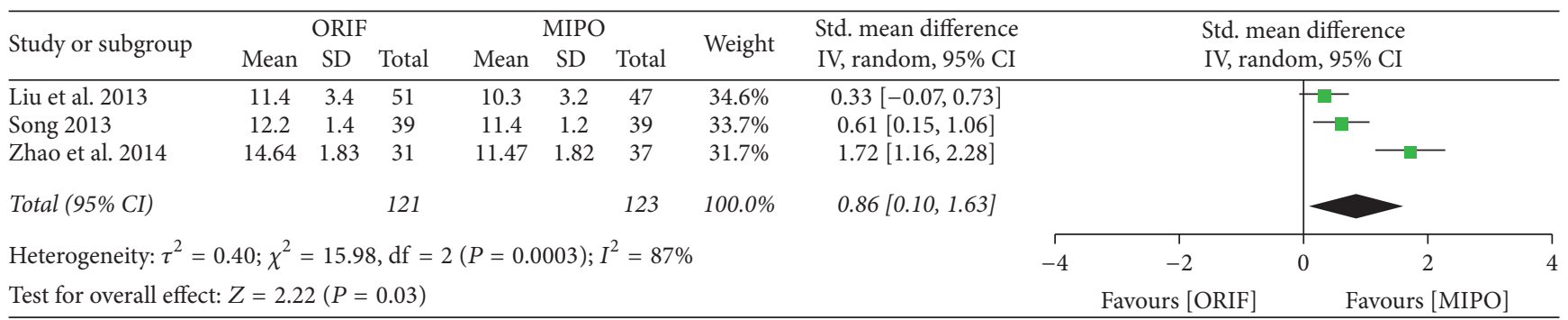

FIGURE 7: Forest plot of standard mean difference (SMD) and 95\% confidence intervals (CI) for fracture healing time.

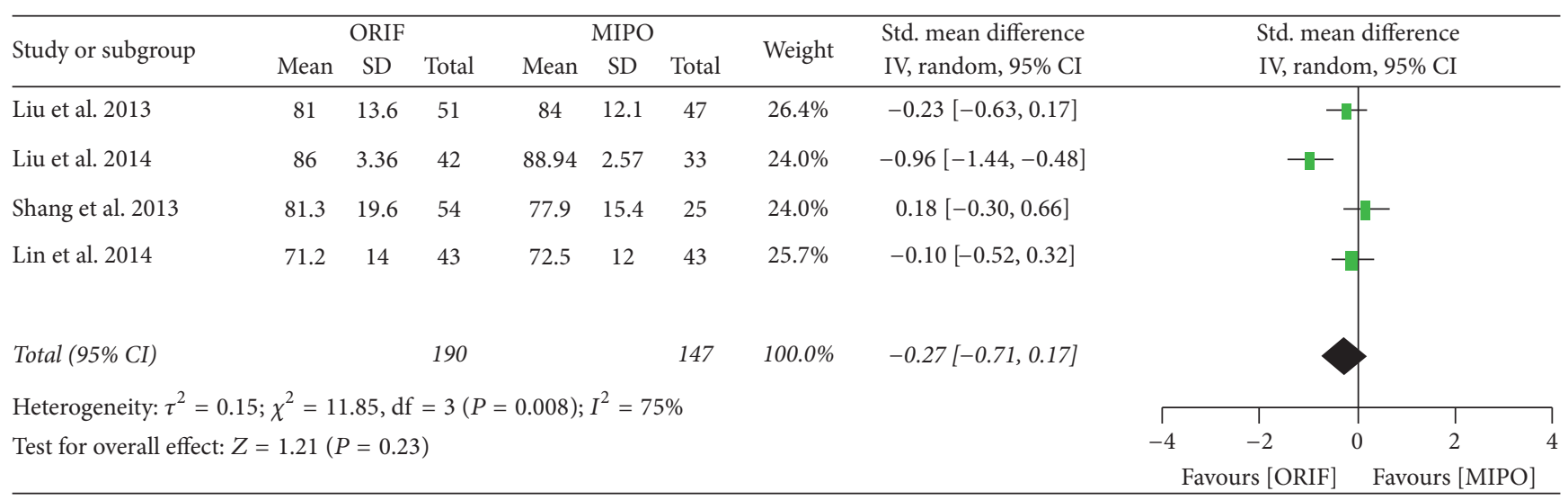

Figure 8: Forest plot of standard mean difference (SMD) and 95\% confidence intervals (CI) for functional outcomes of Constant-Murley score.

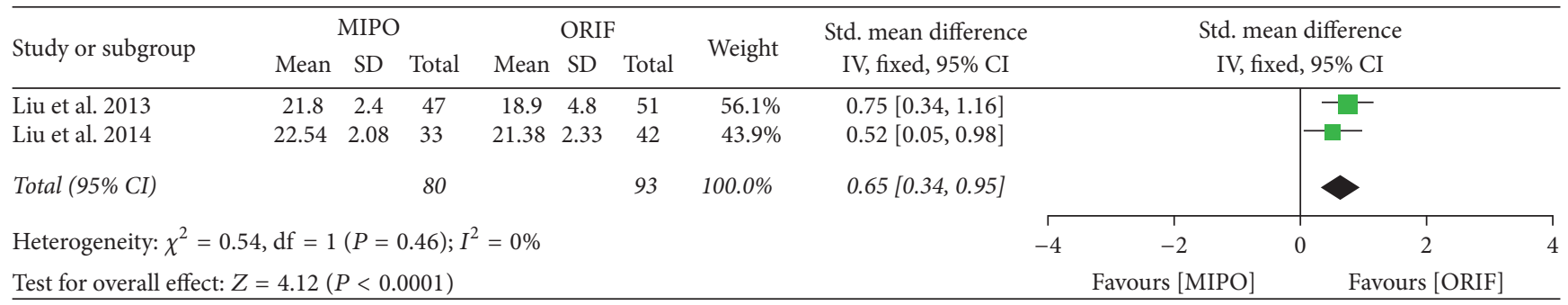

FIGURE 9: Forest plot of standard mean difference (SMD) and 95\% confidence intervals (CI) for muscle strength of Constant-Murley score.

difference between MIPO and ORIF with regard to overall complications. It means that MIPO has minimal risk of operation and is a more appropriate and safer surgical option for elder patients. A shorter operation time and a minimum volume of blood loss could promote postoperative recovery. Furthermore, MIPO treatment for proximal humeral fracture can reduce postoperative pain according to the meta-analysis. An earlier and more effectively functional training would play a positive role for the recovery of motor function. What is more, MIPO had a better outcome of muscle strength scores after operation, enabling patients to engage in early activities and have a better functional exercise. Although these results were obtained, a consensus among orthopedic surgeons on the best treatment for proximal humeral fracture has not been determined $[13,14]$. With regard to a range of surgical managements for proximal humeral fracture, the most important goal of the treatment is to restore a normal function without pain at the shortest time. Improvement of functional outcomes and reduction of postoperative pain are the two most considerable aspects in clinical decision.

ORIF technique for proximal humeral fracture is a traditional method by using deltopectoral approach to the proximal humerus. It provides limited access to the posterolateral aspect of the shoulder, and the visualization and reduction of a large retracted greater tuberosity fragment may be difficult $[15,16]$. The deltopectoral approach requires extensive soft tissue dissection and muscle retraction to gain adequate exposure to the lateral aspect of humerus. It might increase risks on damage of blood supply to humerus head, and thus the incidence of avascular necrosis could be increased [15, 17]. Gardner and colleagues [17] demonstrated preservation of the humeral head arterial supply with a cadaveric study of MIPO, 
including the ascending branch of the anterior humeral circumflex vessel and an unnamed posterior branch $[5,18]$. Lots of clinical studies have demonstrated the superiority of MIPO to ORIF in preventing the damage of blood supply of the humeral head [19]. Shang et al.s study [4] suggested that lateral incisions of MIPO provided less soft tissue dissection when the surgery attained reduction of the greater tuberosity. This technique also had an advantage of processing indirect tractive reduction without periosteotomy, which prevented incidence of nonunion and avascular necrosis. MIPO could be a safer surgical option for elder patients due to consideration of biological and physiological conditions of elder patients.

The meta-analysis has made strict inclusion and exclusion criteria, but it still had some limitations and bias which may be unavoidable. No RCTs were involved in this metaanalysis. As a result, subjective factors may affect the result. Different doctors and different hospitals had a variety of surgical technologies and conditions, which may cause bias. The number of included studies and the data for metaanalysis were limited which frustrated the final results to a certain degree. More rigorous designs and large RCTs are required to make further verification.

In conclusion, the pooled results showed that MIPO was superior to ORIF in the treatment of proximal humeral fracture in elder patients. It was reflected in reducing blood loss, operation time, postoperative pain, or fracture healing time of the surgery and in improving recovery of muscle strength. The MIPO was more suitable than ORIF for treating proximal humeral fracture in elder patients.

\section{Conflicts of Interest}

The authors declare that there are no conflicts of interest regarding the publication of this paper.

\section{Acknowledgments}

This work has been supported by Natural Science Foundation of Liaoning Province of China (201602837).

\section{References}

[1] J. Dai, Y. Chai, C. Wang, and G. Wen, "Meta-analysis comparing locking plate fixation with hemiarthroplasty for complex proximal humeral fractures," European Journal of Orthopaedic Surgery and Traumatology, vol. 24, no. 3, pp. 305-313, 2014.

[2] G. B. Yan, "Neer Classification of proximal humeral fracture," Chinese Journal of Joint Surgery, vol. 2, article 267, 2011.

[3] T. Lin, B. Xiao, X. Ma, D. Fu, and S. Yang, "Minimally invasive plate osteosynthesis with a locking compression plate is superior to open reduction and internal fixation in the management of the proximal humerus fractures," BMC Musculoskeletal Disorders, vol. 15, no. 1, article 206, 2014.

[4] L. P. Shang, F. Zhou, H. Q. Ji, and Z. S. Zhang, "Comparison of curative effects between minimally invasive locking plate internal fixation and open reduction with internal fixation for the treatment of proximal humerus fractures," Journal of Peking Unviersity, vol. 45, no. 5, pp. 711-716, 2013.
[5] T. M. Gregory, E. Vandenbussche, and B. Augereau, "Surgical treatment of three and four-part proximal humeral fractures," Orthopaedics \& Traumatology, Surgery \& Research, vol. 99, no. 1, pp. S197-S207, 2013.

[6] R. Götz, E. Johannes, K. Markus et al., "Second generation locked plating of proximal humerus fractures-a prospective multicentre observational study," International Orthopaedics, vol. 35, no. 3, pp. 425-432, 2011.

[7] Y. W. Liu, X. E. Wei, N. Y. Gao et al., "Case-control study on close manipulative reduction combined with minimally invasive percutaneous plate fixation for the treatment of proximal humeral fractures," China Journal Orthop Trauma, vol. 27, no. 4, pp. 311-315, 2014.

[8] J. Liu, S. H. Li, Z. H. Li et al., "Case-control study on minimally invasive percutaneous new plate osteosynthesis applied in proximal humerus fractures in elder patients," China Journal Orthop Trauma, vol. 26, no. 1, pp. 4-8, 2013.

[9] J. F. Wang, H. B. Song, H. J. Gu, Z. D. Ling, and H. H. Ma, "Casecontrol study on minimally invasive plate osteosynthesis for the treatment of proximal humeurs fractures in elderly patients," China Journal of Orthopaedics and Traumatology, vol. 25, no. 6, pp. 487-489, 2012.

[10] Y. Song, "Two surgical treatments of humeral proximal fractures in the elderly patients," Chinese Journal of Medicinal Guide, vol. 15, no. 4, pp. 597-600, 2013.

[11] D. Q. Zhao, L. N. Zhang, G. Z. Yang et al., "Clinical outcome of osteoporotic proximal humerus fractures treated by minimally invasive percutaneous plate osteosynthesis with use of the proximal humerus internal locking system," Orthopedic Journal of China, vol. 22, no. 6, pp. 508-511, 2014.

[12] G. Wang, Z. Mao, L. Zhang et al., "Meta-analysis of locking plate versus intramedullary nail for treatment of proximal humeral fractures," Journal of Orthopaedic Surgery and Research, vol. 10, no. 1, article 122, 2015.

[13] D. Matziolis, M. Kaeaeb, S. S. Zandi, C. Perka, and S. Greiner, "Surgical treatment of two-part fractures of the proximal humerus: comparison of fixed-angle plate osteosynthesis and Zifko nails," Injury, vol. 41, no. 10, pp. 1041-1046, 2010.

[14] S. Ruchholtz, C. Hauk, U. Lewan, D. Franz, C. Kühne, and R. Zettl, "Minimally invasive polyaxial locking plate fixation of proximal humeral fractures: a prospective study," Journal of Trauma, vol. 71, no. 6, pp. 1737-1744, 2011.

[15] P. Hepp, J. Theopold, C. Voigt, T. Engel, C. Josten, and H. Lill, "The surgical approach for locking plate osteosynthesis of displaced proximal humeral fractures influences the functional outcome," Journal of Shoulder and Elbow Surgery, vol. 17, no. 1, pp. 21-28, 2008.

[16] M. J. Gardner, M. H. Griffith, J. S. Dines, S. M. Briggs, A. J. Weiland, and D. G. Lorich, "The extended anterolateral acromial approach allows minimally invasive access to the proximal humerus," Clinical Orthopaedics and Related Research, no. 434, pp. 123-129, 2005.

[17] M. J. Gardner, J. E. Voos, T. Wanich, D. L. Helfet, and D. G. Lorich, "Vascular implications of minimally invasive plating of proximal humerus fractures," Journal of Orthopaedic Trauma, vol. 20, no. 9, pp. 602-607, 2006.

[18] Y. P. Acklin and C. Sommer, "Plate fixation of proximal humerus fractures using the minimally invasive anterolateral delta split approach," Operative Orthopadie und Traumatologie, vol. 24, no. 1, pp. 61-73, 2012.

[19] C. M. Robinson, L. Khan, A. Akhtar, and R. Whittaker, "The extended deltoid-splitting approach to the proximal humerus," Journal of Orthopaedic Trauma, vol. 21, no. 9, pp. 657-662, 2007. 


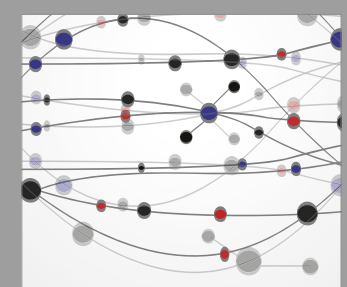

The Scientific World Journal
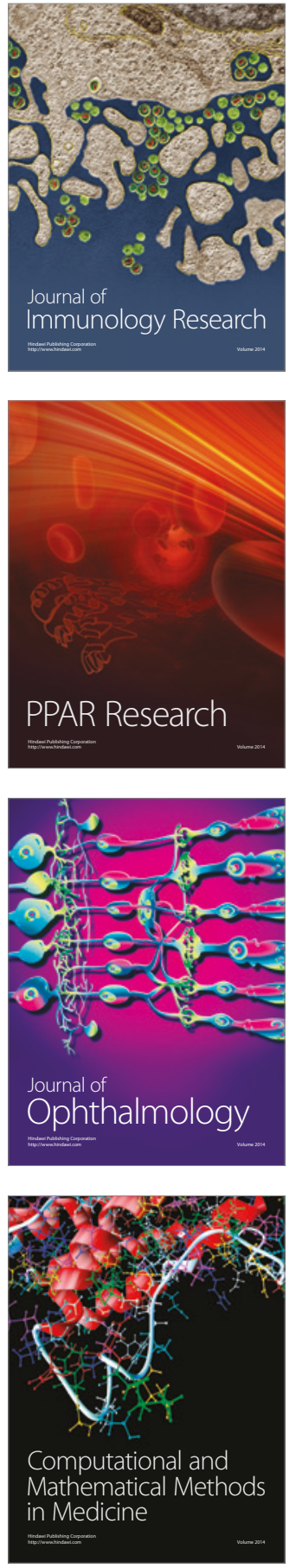

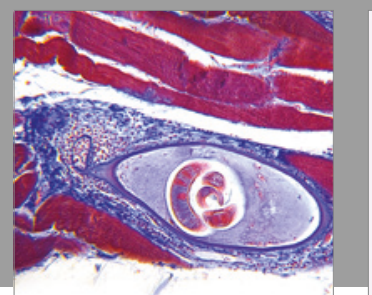

Gastroenterology Research and Practice
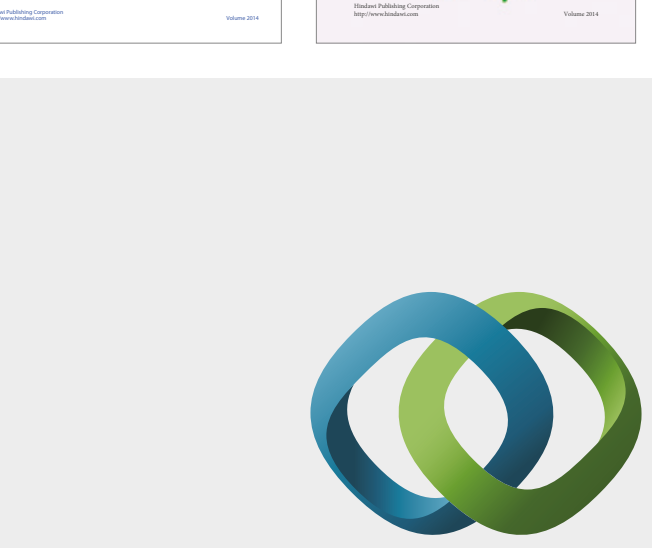

\section{Hindawi}

Submit your manuscripts at

https://www.hindawi.com
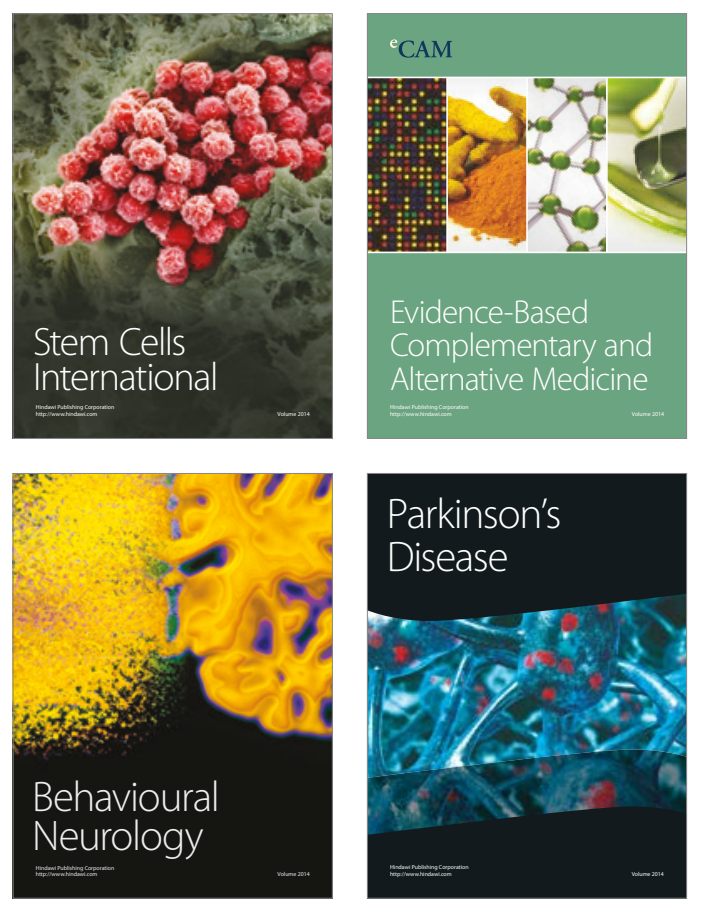
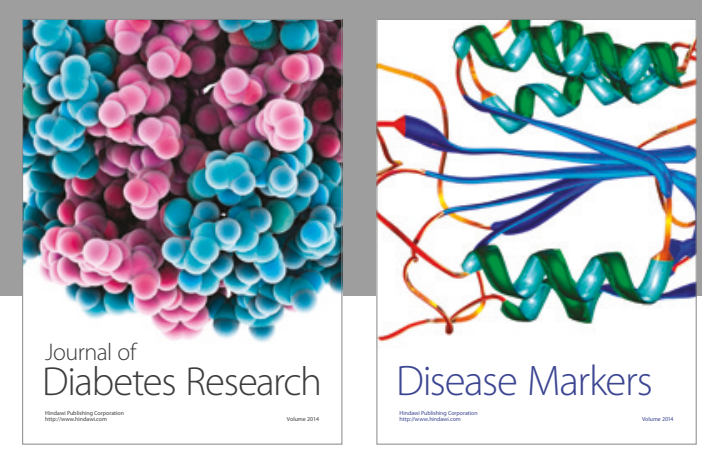

Disease Markers
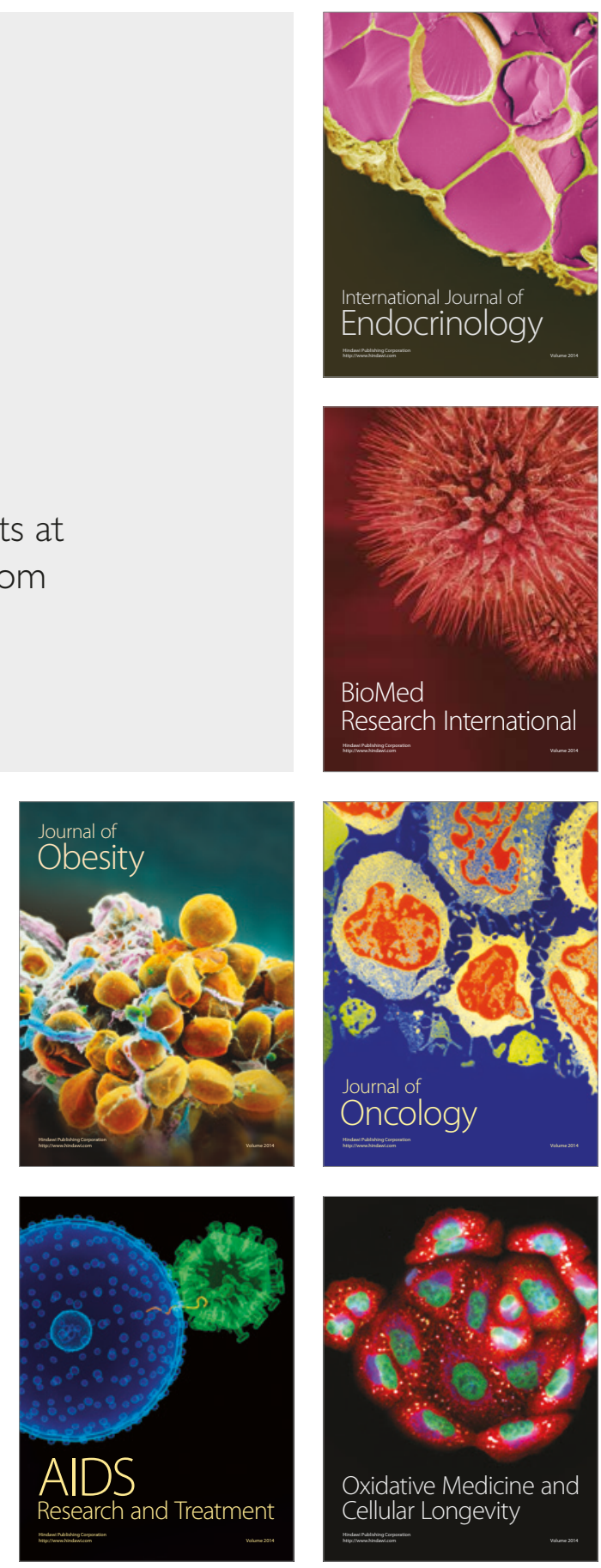